\title{
Single top quark production at CMS
}

\author{
Jose Enrique Palencia Cortezon* on behalf of the CMS collaboration \\ Universidad de Oviedo (Spain) \\ E-mail: palencia@cern.ch
}

\begin{abstract}
The most recent measurements of single top quark production in proton-proton collisions at the LHC at centre-of-mass energies of 7, 8 and $13 \mathrm{TeV}$, using data collected with the CMS experiment, are presented. Precise measurements of the single top quark production cross section in the two most dominant production modes ( $\mathrm{t}$ channel and $\mathrm{tW}$ production), measurements of rare processes involving a top quark and a neutral electroweak boson ( $\mathrm{Z}$ or photon), and searches for flavour changing neutral currents are discussed.
\end{abstract}

Sixth Annual Conference on Large Hadron Collider Physics (LHCP2018)

4-9 June 2018

Bologna, Italy

${ }^{*}$ Speaker. 


\section{Introduction}

Single top quarks, observed for the first time by the D0 [1] and CDF [2] Collaborations at the Fermilab Tevatron, are produced via the electroweak interaction. There are three main production modes in proton-proton ( $\mathrm{pp}$ ) or proton-antiproton collisions: the exchange of a virtual $\mathrm{W}$ boson $(\mathrm{t}$ channel), the production and decay of a virtual $\mathrm{W}$ boson (s channel), and the associated production of a top quark and a W boson (tW channel). These processes are illustrated in Fig. 1.
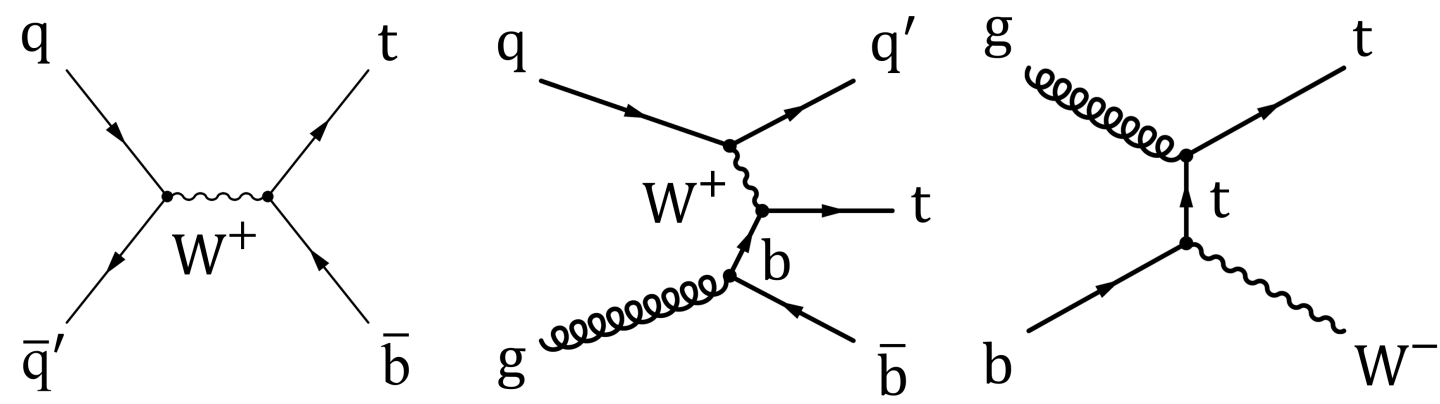

Figure 1: Representative Feynman diagram for electroweak single top quark production in the s channel (left), the dominant next-to-leading-order diagram in the t channel (middle), and the tW production channel (right).

These production modes provide a unique way to probe the Standard Model (SM). In the $\mathrm{t}$ channel, properties of the top quark production, such as the top quark polarization or the CabibboKobayashi-Maskawa (CKM) matrix element $V_{\mathrm{tb}}$, can be probed and parton density functions (PDFs) can be constrained. Measurements of the tW inclusive cross section are particularly interesting to understand interferences with the top quark pair $(\mathrm{t} \overline{\mathrm{t}})$ production mode. Searches for rare SM processes, such as the s channel or the associated production of a single top quark with a $\mathrm{Z}$ boson or a photon, are particularly relevant for testing the SM, but are also experimentally challenging. Single top quark measurements can be used to search for physics beyond the SM (BSM) because they are backgrounds to new physics signals or because they are sensitive to BSM contributions.

This document provides a review of the current experimental studies of processes with a single top quark with the CMS detector [3] at the LHC pp collider at different centre-of-mass energies.

\section{2. t channel production}

The production of single top quarks provides a unique testing ground for the study of electroweak processes, specifically the tWb vertex, as well as the measurement of the CKM matrix element $V_{\mathrm{tb}}$. This decay mode represents $\approx 73 \%$ of the total single top quark production at the LHC and presents a distinct signature, a light quark recoiling against the top quark. Due to its relative large production rate, differential measurements are possible in this channel.

The cross section for the production of single top quarks in the t channel is measured at $\sqrt{s}$ $=13 \mathrm{TeV}$ [4]. The analyzed data correspond to an integrated luminosity of $2.2 \mathrm{fb}^{-1}$. The event 
selection requires one muon and two jets where one of the jets is identified as originating from a bottom quark. Several kinematic variables are then combined into a multivariate discriminator to distinguish signal from background events. The distribution of this discriminator is shown in Fig. 2 (left). A fit to the distribution of the discriminating variable yields a total cross section of $238 \pm 13$ (stat) \pm 12 (exp) \pm 26 (theo) \pm 5 (lumi) pb and a ratio of top quark and top antiquark production of $\mathrm{R}_{\mathrm{t}-\mathrm{ch}}=1.81 \pm 0.18$ (stat) \pm 0.15 (syst). From the total cross section the absolute value of the CKM matrix element $V_{\mathrm{tb}}$ is calculated to be $1.05 \pm 0.07$ (exp) \pm 0.02 (theo). All results are in agreement with the SM predictions.
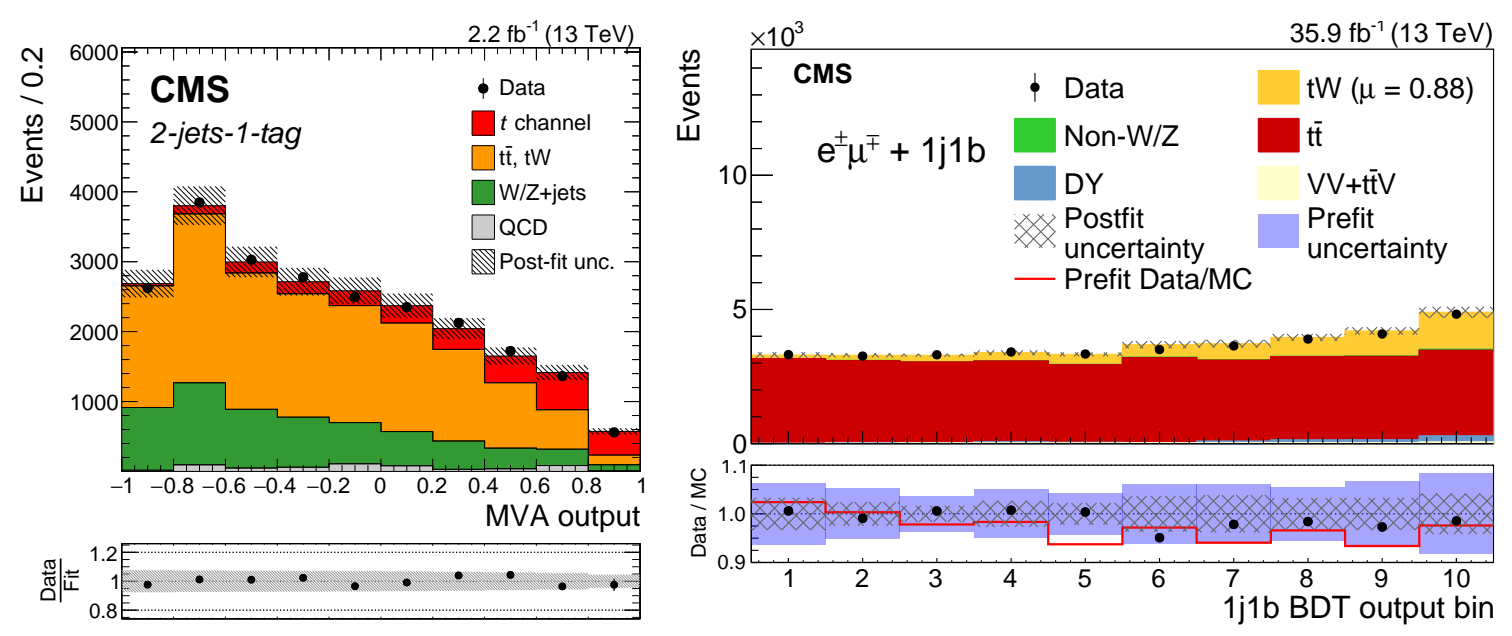

Figure 2: Left: Multivariate discriminator distributions for all muons normalized to the yields obtained from the simultaneous fit in the 2-jets-1-tag region. The hatched areas indicate the post-fit uncertainties [4]. Right: Comparison of the BDT output in the $1 \mathrm{j} 1 \mathrm{~b}$ region, after the fit is performed for the observed data and simulated events. The error band includes the statistical and systematic uncertainties. The bottom of the panel shows the ratios of data to the prediction from simulations and from the fit [5].

\section{3. tW production}

The $\mathrm{tW}$ process provides a unique opportunity to study the SM and its extensions through the interference of the process at next-to-leading order (NLO) with ț production [6]. The tW process also plays an important role because of its sensitivity to the physics BSM [7].

The data recorded at $\sqrt{s}=13 \mathrm{TeV}$, corresponding to an integrated luminosity of $35.9 \pm 0.9$ $\mathrm{fb}^{-1}$, are used to measure the tW production cross section in the e $\mu$ channel [5], classifying the events in terms of the number of jets and jets originating from bottom quarks. The signal is measured using a maximum-likelihood fit to the distribution of boosted decision tree (BDT) discriminants in two of the categories, and to the $\mathrm{p}_{T}$ distribution of the second jet with highest $\mathrm{p}_{T}$ in a third category. The distribution of the BDT output in the most signal-enriched region is shown in Fig. 2 (right). The measured cross section for tW production is found to be $63.1 \pm 1.8$ (stat) \pm 6.4 (syst) \pm 2.1 (lumi) pb, achieving a relative uncertainty of $11 \%$. The measured cross section is in agreement with the $\mathrm{SM}$ prediction of $71.7 \pm 1.8$ (scale) \pm 3.4 (PDF) pb. 


\section{4. s channel production}

The s channel production is a rare process, only $\approx 3 \%$ of the total single top quark events produced at the LHC. On top of that, its production rate grows much lower with the centre-of-mass energy than other top quark production modes. It is of special interest since a possible deviation from the SM prediction of its cross section may indicate the presence of BSM processes, as predicted by models that involve the exchange of a non-SM mediator, such as a $\mathrm{W}$ boson or a charged Higgs boson [8].

A search for single top quark production in the s channel at centre-of-mass energies of 7 and $8 \mathrm{TeV}$ is presented [9]. Decay modes of the top quark containing a muon or an electron in the final state are used. A multivariate approach based on BDTs is adopted to discriminate the signal from background contributions. The signal is extracted through a maximum-likelihood fit to these distributions. The cross section is measured to be $7.1 \pm 8.1$ (stat+syst) pb at $\sqrt{s}=7 \mathrm{TeV}$ and $13.4 \pm 7.3$ (stat+syst) pb at $\sqrt{s}=8 \mathrm{TeV}$, corresponding to a combined signal rate relative to $\mathrm{SM}$ expectations of $2.0 \pm 0.9$ (stat+syst). The observed and expected upper limits on the combined signal strength are found to be 4.7 and 3.1 at $95 \%$ confidence level, respectively. The observed significance of the combined measurement is 2.5 standard deviations with 1.1 standard deviations expected. The measurements are in agreement with the prediction of the SM.

\section{5. $\mathrm{tZ}$ production}

The associated production of a single top quark with a $\mathrm{Z}$ boson is a rare process with a very small cross section, $\sim 2$ orders of magnitude smaller than the tW production. This process is sensitive to top quark couplings to the $\mathrm{Z}$ boson and also to the triple gauge-boson coupling WWZ.

The associated production cross section of a single top quark and a $\mathrm{Z}$ boson was measured using data from pp collisions at $\sqrt{s}=13 \mathrm{TeV}$, corresponding to an integrated luminosity of 35.9 $\mathrm{fb}^{-1}$ [10]. The measurement uses events containing three charged leptons in the final state and is based on a multivariate analysis, where BDTs are used to enhance the signal-to-background separation. The distributions used for the signal extraction are shown in Fig. 3. Several control regions are defined to better constrain the backgrounds, each containing different contributions from signal and background processes. Evidence for tZq production is found with an observed (expected) significance of 3.7 (3.1) standard deviations. The cross section is measured to be $123_{-31}^{+33}$ (stat) ${ }_{-23}^{+29}$ (syst) $\mathrm{fb}$, for $\mathrm{m}_{\ell \ell}>30 \mathrm{GeV}$, where $\ell$ stands for electrons, muons, or $\tau$ leptons. This value is compatible with the NLO SM prediction of $94.2 \pm 3.1 \mathrm{fb}$.

\section{6. $\mathrm{t} \gamma$ production}

The study of top quark production in association with a photon is an important test of the SM description of top quark interactions with gauge bosons, and plays an important role in the search for physics BSM. The cross section for single top quark production in association with a photon is sensitive to the top quark charge, and to its electric and magnetic dipole moments [11, 12].

The first evidence of events consistent with the production of a single top quark in association with a photon is reported. The analysis is based on pp collisions at $\sqrt{s}=13 \mathrm{TeV}$, corresponding 

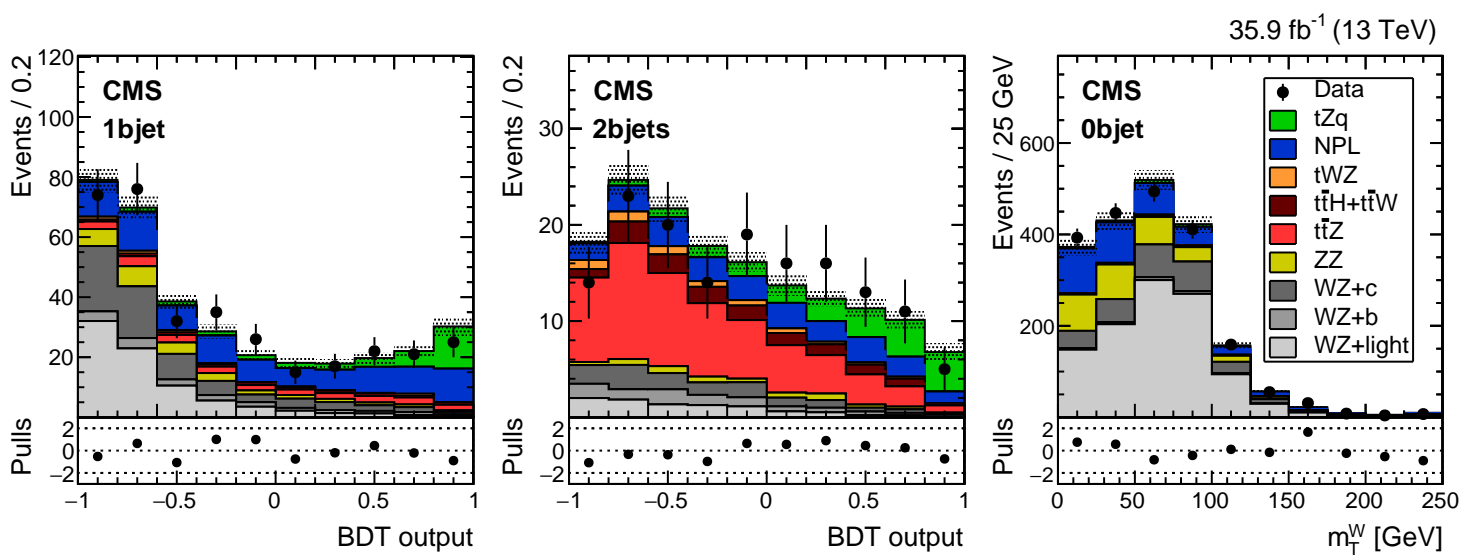

Figure 3: Template distributions used for signal extraction [10]. Left: BDT discriminator in the $1 \mathrm{~b}$-tagged jet region; centre: BDT output in the 2 b-tagged jets control region; right: $\mathrm{W}$ boson transverse mass in the objet control region. The predictions correspond to the normalizations obtained after the fit. The hatched bands include the total uncertainty on the background and signal contributions. The pulls in the distributions are shown in the bottom panels.

to an integrated luminosity of $35.9 \mathrm{fb}^{-1}$ [13]. Events are selected by requiring the presence of a muon, a photon, an imbalance in transverse momentum from an undetected neutrino, and at least two jets of which exactly one is identified as associated to the hadronization of a $b$ quark. A multivariate discriminant based on topological and kinematic event properties is employed to separate signal from background processes. In order to extract the signal cross section and the background normalization, a simultaneous binned likelihood fit is performed on the BDT distribution in the signal and control regions. The distributions used for the signal extraction after the fit are shown in Fig. 4 (left). An excess above the background-only hypothesis is observed, with a significance of 4.4 standard deviations. A fiducial cross section is measured for isolated photons with transverse momentum greater than $25 \mathrm{GeV}$ in the central region of the detector. The measured product of the cross section and branching fraction is $115 \pm 17$ (stat) \pm 30 (syst) fb, which is consistent with the SM prediction.

\section{Flavour changing neutral currents}

In the SM, flavour changing neutral currents (FCNC) are forbidden at tree level and highly suppressed at higher order, resulting in a SM branching fraction for a top quark decaying into a charm or up quark and a $\mathrm{Z}$ boson of the order of $10^{-14}[14,15]$. Several extensions of the SM enhance the FCNC branching fractions and can be probed at the LHC [14]; the new couplings can also provide for flavour changing single top quark production in association with a $\mathrm{Z}$ boson.

A search for FCNC involving a top quark and a $\mathrm{Z}$ boson is done using the proton collision data at a centre-of-mass energy of $13 \mathrm{TeV}$, corresponding to an integrated luminosity of $35.9 \mathrm{fb}^{-1}$ [16]. The search focuses on single top quark and top quark pair FCNC interactions observable in three lepton final states, where the FCNC interaction happens at the production or at the top quark decay. As an aid in classifying events as signal or background, two multivariate discriminants based on BDTs are employed, one targeting the single top quark production signal and the other the top 

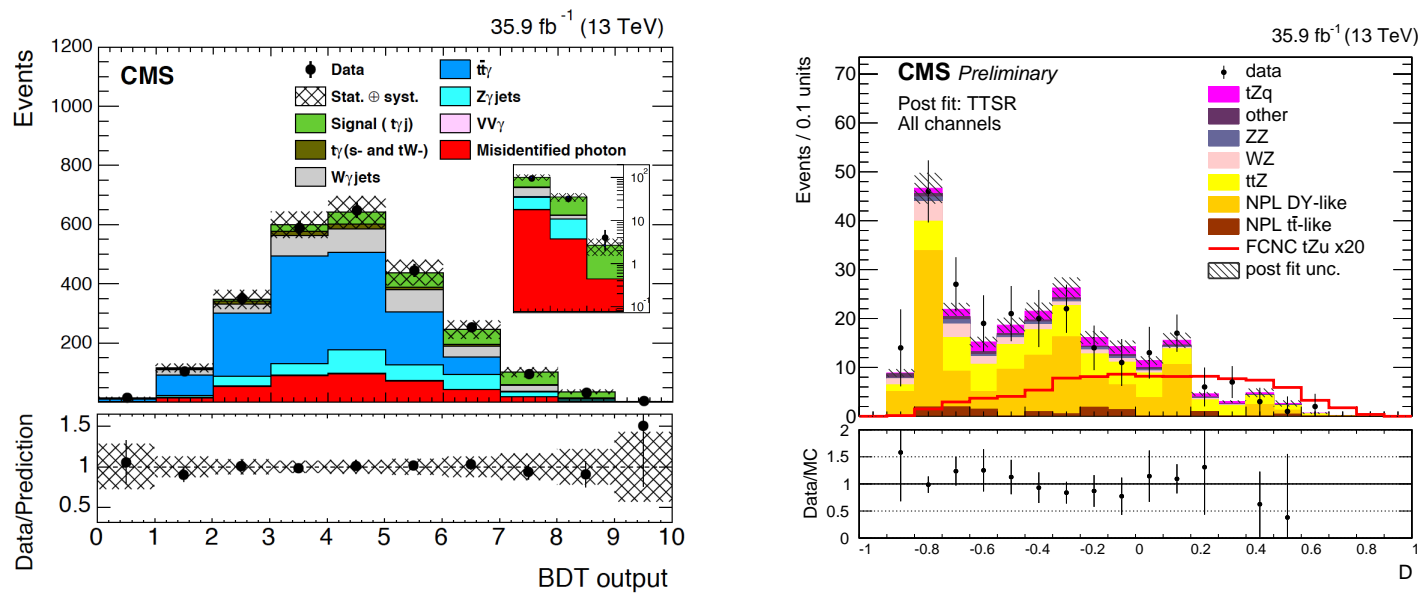

Figure 4: Left: The BDT output distribution for data and SM predictions after performing the fit [13]. The inset presents a zoom of the last three bins plotted on log scale. The hatched band shows the statistical and systematic uncertainties in the estimated signal and background yields. Right: The discriminating variable distribution after the fit for all different leptonic channels in the $t \bar{t}$ signal region [16].

quark decay signal. Four different lepton channels based on lepton flavour are considered. The analysis strategy uses five statistically independent regions to extract limits using a likelihood fit of various observables. A simultaneous global fit is performed taking into account each one of the five regions for the four different leptonic channels. The distribution of the BDT output after the fit for all different leptonic channels is shown in Fig. 4 (right). No significant deviation is observed from the predicted background. Observed (expected) upper limits at 95\% confidence level are set on the branching fractions of top quark decays: $\mathrm{B}(\mathrm{t} \rightarrow \mathrm{uZ})<0.024 \%(0.015 \%)$ and $\mathrm{B}(\mathrm{t} \rightarrow \mathrm{cZ})<0.045 \%$ $(0.037 \%)$, assuming one non-vanishing FCNC coupling at a time.

\section{Summary}

CMS has measured production rates of processes with a single top quark in five production modes, at three distinct centre-of-mass energies. Precise measurements of the single top quark production cross section in the two most dominant production modes ( $\mathrm{t}$ channel and tW production), measurements of rare processes involving a top quark and a neutral electroweak boson ( $\mathrm{Z}$ or photon), and searches for flavour changing neutral currents have been discussed. A summary of the different measurements is shown in Fig. 5.

\section{References}

[1] D0 Collaboration, Observation of Single Top Quark Production, Phys. Rev. Lett. 103 (2009) 092001 [arXiv:0903.0850].

[2] CDF Collaboration, First Observation of Electroweak Single Top Quark Production, Phys. Rev. Lett. 103 (2009) 092002 [arXiv:0903.0885].

[3] CMS Collaboration, The CMS Experiment at the CERN LHC, JINST 3 S8004 (2008). 


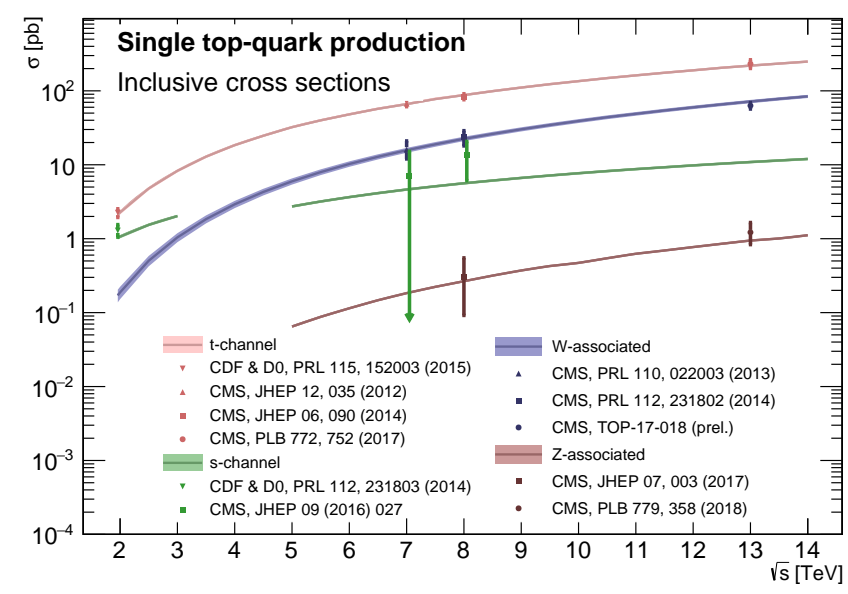

Figure 5: Summary of the single top quark cross section measurements by CMS, as function of centre-ofmass energy.

[4] CMS Collaboration, Cross section measurement of t-channel single top quark production in pp collisions at $\sqrt{s}=13 \mathrm{TeV}$, Phys. Lett. B 772 (2017) 752 [arXiv:1610.00678].

[5] CMS Collaboration, Measurement of the production cross section for single top quarks in association with $W$ bosons in proton-proton collisions at $\sqrt{s}=13 \mathrm{TeV}$, arXiv:1805.07399.

[6] S. Frixione et al., Single-top hadroproduction in association with a W boson, JHEP 07 (2008) 029.

[7] T. M. P. Tait and C. P. Yuan, Single top quark production as a window to physics beyond the standard model, Phys. Rev. D 63 (2000) 014018 [arXiv:hep-ph/0007298].

[8] M. Hashemi, Observability of heavy charged Higgs through s-channel single top events at LHC, JHEP 11 (2013) 005 [arXiv:1310.5209].

[9] CMS Collaboration, Search for s channel single top quark production in pp collisions at $\sqrt{s}=7$ and 8 TeV, JHEP 09 (2016) 027 [arXiv:1603.02555].

[10] CMS Collaboration, Measurement of the associated production of a single top quark and a $Z$ boson in pp collisions at $\sqrt{s}=T e V$, Phys. Lett. B 779 (2018) 358 [arXiv:1712.02825].

[11] M. Fael and T. Gehrmann, Probing top quark electromagnetic dipole moments in single-top-plus-photon production, Phys. Rev. D 88 (2013) 033003, [arXiv:1307.1349].

[12] S. M. Etesami, S. Khatibi, and M. Mohammadi Najafabadi, Measuring anomalous WW $\gamma$ and $t \bar{t} \gamma$ couplings using top $+\gamma$ production at the LHC, Eur. Phys. J. C 76 (2016) 533 [arXiv:1606.02178].

[13] CMS Collaboration, Evidence for the associated production of a single top quark and a photon in proton-proton collisions at $\sqrt{s}=13 \mathrm{TeV}$, arXiv:1808.02913.

[14] J. A. Aguilar-Saavedra, Top flavor-changing neutral interactions: Theoretical expectations and experimental detection, Acta Phys. Polon. B (2004) 2695 [arXiv:hep-ph/0409342].

[15] S. L. Glashow, J. Iliopoulos, and L. Maiani, Weak interactions with lepton-hadron symmetry, Phys. Rev. D 2 (1970) 1285.

[16] CMS Collaboration, Search for flavour changing neutral currents in top quark production and decays with three-lepton final state using the data collected at $\sqrt{s}=13 \mathrm{TeV}$, CMS-PAS-TOP-17-017 [https://cds.cern.ch/record/2292045]. 